\title{
Liturgie - die bessere Show? Das „Medienreligiöse" als Herausforderung an die Kirchen ${ }^{1}$
}

\author{
von Armo Schilson
}

Die Antwort auf die Titelfrage dieses Beitrags scheint problemlos und einfach. Wer die Liturgie mit einer Show vergleichen will, wer sie mit einer als seicht empfundenen Fernsehunterhaltung auf eine Ebene stellt, der hat offenbar ihre ureigene Würde und ihren ganz anderen Charakter völlig verkannt. Er zieht sie herab auf eine Ebene, die ihr nie und nimmer gerecht werden kann.

Daß diese allzu rasche und glatte Antwort der Sache selbst kaum entspricht, zeigt sich bereits bei einer ersten kritischen Rückfrage. Immerhin ist die Liturgie ihrer Grundgestalt und ihrer Natur nach einer Show nicht so absolut unähnlich und damit so unvergleichbar, wie es zunächst scheinen mag. Oft wird vergessen, daß christliche Liturgie zwar als eine heilige Handlung, aber eben doch als eine Handlung zu betrachten ist. Man hat sie nicht zu Unrecht auch Drama und heiliges Theater, Schauspiel genannt.2 Liturgie ist "action" - und mit diesem

Dr. theol. habil. Arno Schilson ist Professor für Abendländische Religionsgeschichte der Johannes-Gutenberg-Universität Mainz.

1 Die folgenden Überlegungen dokumentieren einen Vortrag, der bei einer von der Thomas-Morus-Akademie Bensberg in Zusammenarbeit mit der Zentralstelle Medien der Deutschen Bischofskonferenz zum Thema "Das Religiöse im Säkularen. Werbung, Musikvideos, Unterhaltungsshows" im Dezember 1995 veranstalteten Studienkonferenz gehalten wurde. Ausdrücklich zu danken habe ich Herrn Dr. Gregor Taxacher von der Akademie in Bensberg, der mir die Dringlichkeit dieses Themas deutlich gemacht hat.

Entscheidende Anregungen und Denkanstöße für die folgenden Überlegungen verdanke ich vor allem Horst Albrecht, Die Religion der Massenmedien, Stuttgart - Berlin - Köln 1993 (Lit.); Eckhard Bieger u.a. (Hg.), Zeitgeistlich. Religion und Fernsehen in den neunziger Jahren, Köln - Bonn 1993; Hartmut Heuermann, Medienkultur und Mythen. Regressive Tendenzen im Fortschritt der Moderne, Reinbek 1994; Hans-Joachim Höhn, Gegen-Mythen. Religionsproduktive Tendenzen der Gegenwart, Freiburg - Basel - Wien 1994. Ergänzend vgl. auch: Katholische Fernseharbeit beim ZDF (Hg.), Die Kirche wickelt sich ab - und die Gesellschaft lebt die produktive Kraft des Religiösen, Mainz 21995.

2 Das bleibt eine der bis heute wirksamsten und bedeutendsten Entdeckungen des großen Theologen Odo Casel; vgl. dazu meine Interpretationen: Theologie als Sakramententheologie. Die Mysterientheologie Odo Casels, Mainz 21987; Die Gegenwart des Ursprungs. Überlegungen zur bleibenden Aktualität der Mysterientheologie Odo Casels, in: Liturgisches Jahrbuch 43 (1993) 6-29 (Lit.). Ergänzend s.a. zur einschlägigen neueren Lit. meine Berichte: „Feier" und "Heiliges Spiel”. Wandlungen im heutigen Gottes- 
schlichten Fremdwort unserer Tage offenbart sich die ganze Ambivalenz und prinzipielle Unentschiedenheit der Titelfrage. Im Gottesdienst geschieht etwas, das (zumindest grundsätzlich) faszinierend, mitreißend und begeisternd wirkt, das Menschen über den Alltag erhebt und sie irgend wie beglückt. $\mathrm{Ob}$ das allein schon genügen kann, um die Liturgie als Show oder gar als die "bessere Show" zu betrachten, sei zunächst einmal dahingestellt. Immerhin finden sich in der Liturgie durchaus weitere Elemente und Strukturen, die ebenso jeder Fernsehshow eignen: Ein klarer und durchsichtiger Aufbau mit verteilten Rollen, Haupt- und Nebenakteure, feststehende Rituale (etwa Begrüßung und Verabschiedung, Präsentation und Erläuterung besonderer Elemente u.ä.), reichlich Musik und die aktive Einbeziehung der Anwesenden - all das und vieles mehr läßt sich durchaus vergleichen.

Doch eine solche Betrachtung und ein solcher Vergleich greifen zu kurz. Wer nur die äußere Form der Zusammenkunft und deren Strukturen betrachtet, findet zwar durchaus ähnliches. Er hat jedoch die viel tieferen Dimensionen noch nicht erfaßt, die das Medium Fernsehen in die religiöse Szene unserer Tage einbringt und worauf die Kirchen und ihre Theologie nicht nur strukturell, sondern inhaltlich in engagierter Wahrnehmung und kritischer Auseinandersetzung zu reagieren haben. Als Herausforderung an die Kirchen soll dieses noch reichlich

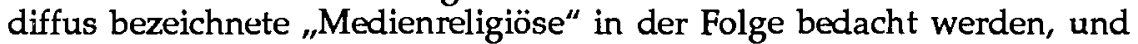
zwar konkretisiert und zugespitzt auf das Geschehen der Liturgie, dem in der heutigen Situation der Kirche (zumindest im evangelischen Bereich!) vermehrte Aufmerksamkeit zuteil wird. ${ }^{3} \mathrm{Um}$ hier möglichst

dienst- und Sakramentenverständnis, in: Klemens Richter und Arno Schilson, Den Glauben feiern. Wege liturgischer Erneuerung, Mainz 1989, 78-108, und: Fest und Feier in anthropologischer und theologischer Sicht. Ein Literaturbericht, in: Liturgisches Jahrbuch 44 (1994) 3-32.

3 Außer der in Anm. 2 bereits genannten Lit. vgl. bes. die ebenso anregende wie sorgfältig gearbeitete Studie von Peter Cornehl, Teilnahme am Gottesdienst. Zur Logik des Kirchgangs - Befund und Konsequenzen, in: Joachim Matthes (Hg.), Kinchenmitgliedschaft im Wandel. Untersuchungen zur Realität der Volkskirche. Beiträge zur zweiten EKD-Umfrage „Was wird aus der Kirche?", Gütersloh 21991, 15-53 (Lit.); ergänzend auch die früheren einschlägigen Beiträge ders., Gottesdienst, in: Ferdinand Klostermann - Rolf Zerfaß (Hg.), Praktische Theologie heute, München - Mainz 1974, 449-463; Theorie des Gottesdienstes - ein Prospekt, in: Theologische Quartalschrift 159 (1979) 178-195. Weiteres zur Sache sowie zur Lit. s. in meinen Beiträgen: Liturgie und Menschsein. Überlegungen zur Liturgiefähigkeit des Menschen am Ende des 20. Jahrhunderts, in: Liturgisches Jahrbuch 39 (1989) 206-227; Christlicher Gottesdienst - Ort des Menschseins. Fundamentalliturgische Überlegungen in ökumenischer Absicht, in: Karl Schlemmer (Hg.), Gemeinsame Liturgie in getrennten Kirchen?, Freiburg 1991, 53-81; Das Christentum - eine Mysterienreligion? Geschichtliche und grundsätzliche Hinweise zu einer aktuellen Frage, in: Hermann Kochanek ( $\mathrm{Hg}$.), Heil durch Erfahrung und Erkenntnis. Die Herausforderung von Gnosis und Esoterik für das frühe Christentum und seine Gegenwart, Nettetal 1993, 91-112; Unsere Gottesbe- 
konkret zu werden, empfehlen sich drei eng miteinander verzahnte Gedankenschritte, in denen sich bereits eine übergreifende These andeutet und entfaltet.

An erster Stelle bleibt zu bedenken, welche Momente und Gestalten dem reichlich komplexen Phänomen des Medienreligiösen eignen, worin es sich äußert und was es darstellen soll und will. Dabei wird eine erste Herausforderung für die Kirchen nicht nur sichtbar, sondern dieser zugleich entsprochen: Zunächst gilt es, das Medienreligiöse in seiner vielfältigen Gestalt wahrzunehmen und die sich darin artikulierenden Momente auf ihre spezifisch religiösen Bedürfnisse und Motive hin zu deuten. Nüchternes Wahrnehmen und verstehendes Deuten müssen am Anfang stehen, will man die hier vorliegende Herausforderung in ihrem wahren Umfang begreifen, sie möglicherweise als echte Chance verstehen und so darauf reagieren.

Dem verstehenden Zur-Kenntnis-Nehmen muß natürlich eine kritische Würdigung folgen. Verächtliche Zurückweisung und arrogante Überheblichkeit sind unter dieser Rücksicht völlig verfehlt. Die zahlreichen und gefährlichen Schlagworte von "verwässertem Christentum", "Ausverkauf des Christlichen“, „säkularisiertem Evangelium" oder "verweltlichter Liturgie" sind kaum dazu angetan, diesem speziellen Sektor der religiösen Szene der Gegenwart in seinem besonderem Anspruch an die Kirchen angemessen zu begegnen und ihn zu würdigen. ${ }^{4}$ Bei aller Kritik, die hier not tut und zugleich Hilfe bedeuten kann, gilt es zunächst einmal, den reflektierend wahrgenommenen Phänomenbefund auch ernst zu nehmen und als echte, durchaus positiv aufzugreifende Herausforderung zu verstehen.

Erst wo dieser wichtige Schritt erfolgt und das Medienreligiöse gleichsam mit "kritischer Sympathie“ auf seine Stärken und Schwächen hin durchleuchtet und als eine beachtliche Ausdrucksform gegenwärti-

ziehung in Liturgie und Gebet. Versuch einer Wegweisung, in: Anzeiger für die Seelsorge 103 (1994) 309-314, 368-373; Der Gottesdienst als „Höhepunkt und Quelle" des christlichen Lebens. Herkunft und Bedeutung einer programmatischen Aussage des Konzils, in: Bernd Jochen Hilberath und Dorothea Sattler $(\mathrm{Hg}$.$) , Vorgeschmack. Ökumenische Bemühungen um die$ Eucharistie. Festschrift für Theodor Schneider, Mainz 1995, 293-307. Anregend auch Gerhard Marcel Martin, Ausverkauf oder armes Theater. Unser Kultus im Kontext gegenwärtiger Kultur, in: Zeitschrift für Gottesdienst und Predigt 8 (1990) 31-35.

4 Zum allgemeinen Phänomen heutiger säkularer Religiosität und dessen Herausforderung für die Kirchen vgl. meine Sichtung einschlägiger Lit.: Die religiöse Dimension der Gegenwart, in: Herder-Korrespondenz 50 (1996) 28-34. Ergänzend s.a. die brillante Skizze von Wilhelm Gräb, Auf den Spuren der Religion. Notizen zur Lage und Zukunft der Kirche, in: Zeitschrift für Evangelische Ethik 39 (1995) 43-56 (Lit.), sowie Evangelische Akademie Baden/Katholische Akademie Freiburg (Hg.), Glauben ohne Kirche. Neue Religiosität als Herausforderung für die Kirchen, Freiburg - Karlsruhe 1995. 
ger Religion akzeptiert wird, kann eine fruchtbare Auseinandersetzung damit erfolgen. Dabei darf keineswegs die satte Selbstzufriedenheit dessen vorherrschen, der die Fülle der Wahrheit und deren allein richtige Ausdrucksgestalt zu besitzen meint. Vielmehr gilt es, die unabweisbaren Fragen des Medienreligiösen an die Kirchen und ihre Theologie, vor allem aber an die Liturgie, mit einem gerüttelten Maß an Bereitschaft zur Selbstkritik aufzunehmen und so auch unleugbare Schwächen und Einseitigkeiten, bedauerliche Fehlentwicklungen und Mängel in den heutigen Gottesdiensten der Kirche zu erkennen und auszumerzen. Von Anbiederung sollte und kann dabei keine Rede sein. $\mathrm{Da}$ die christlichen Kirchen mit ihrer Botschaft und mit ihren Gottesdiensten grundsätzlich auch dazu fähig sind und sein müssen, der Herausforderung des Medienreligiösen zu begegnen, die darin enthaltenen, durchaus legitimen und Respekt heischenden Anliegen aufzugreifen und deren Erfüllung in kritischer Überbietung praktisch und anschaulich zu vermitteln, steht dabei außer Frage.

Nachdem so der Prospekt und der Rahmen sowie die leitenden Überzeugungen für die folgenden Überlegungen umrissen sind, soll nun konkreter gesprochen und das Phänomen selbst genauer beleuchtet werden.

\section{Das Medienreligiöse wahrnehmen und deuten}

Die erste und grundlegende Frage muß lauten: Was ist das eigentlich, das Medienreligiöse? Worin und wie zeigt es sich, und welches Grundmoment von Religion läßt sich deutend darin erkennen?5

\subsection{Das Religiöse in der Werbung}

Allbekannt ist mittlerweile, daß die Werbung in reichem Maße sich religiöser Symbolelemente und Anspielungen auf religiöse, im europäischen Kulturkreis dominant christliche Gehalte bedient. ${ }^{6}$ Der Glanz und die Reinheit einer anderen Welt erstrahlen aus jeder Waschmittelreklame und suggerieren: Wenn schon niemand - moralisch betrachtet in seinem Leben ein Saubermann oder eine Sauberfrau sein und bleiben kann, dann verschafft zumindest saubere, reine Wäsche ein Gefühl ursprünglicher Reinheit und Unschuld. Dabei weiß die Werbung sehr

5 Dazu und zum Folgenden vgl. nochmals die in Anm. 1 genannte Lit., bes. Bieger 35-54: Religion in den konkreten Sendeformen; ergänzend auch Angela Keppler, Die Kommunion des Dabeiseins. Formen des Sakralen in der Fernsehunterhaltung, in: Rundfunk und Fernsehen 43 (1995) 301-311.

6 Vgl. dazu außer Albrecht (Anun. 1) 42-62 noch Holger Tremel (Hg.), Das Paradies im Angebot. Religiöse Elemente in der Werbung, Frankfurt am Main 1986, sowie Matthias Wörther, Wer entleert die Symbole? Religiöse und ethische Elemente in der Werbung, in: Katechetische Blätter 120 (1995) 243-246 (Lit.). 
wohl um die qualitative Differenz zwischen schlicht materiell-sichtbarer Sauberkeit und dem sowohl dem kultischen als auch moralischen Bereich zugehörenden und damit entschieden religiös konnotierten Begriff der Reinheit. Gleiches zeigt sich dort, wo von "himmlischem Vergnügen" die Rede ist. Und wenn eine Marmeladenreklame ihre Produkte empfiehlt als "die schönsten Früchte aus dem Garten Eden"7, dann sind die werbewirksam eingesetzten Anleihen aus der christlichen Tradition unverkennbar. Daß neuerdings vermehrt Nonnen oder Kirchenmänner für ein Produkt figurieren, gehört ebenfalls hierher. Immerhin läßt sich daraus soviel erkennen: $\mathrm{Da}$ Werbung ihrem Selbstverständnis nach auf allgemeine Akzeptanz und Überzeugungskraft setzen muß, dokumentiert sich in der Vielzahl (oft latent) religiös geprägter Werbeelemente die Überzeugung, daß solche religiösen Motive und Dimensionen nicht auf Ablehnung, sondern auf breiteste Zustimmung stoßen und damit die erwünschte suggestive Wirkung ausüben.

Doch viel wichtiger als diese einzelnen religiösen Momente in der Werbung ist die Strukturähnlichkeit, die sie ganz grundsätzlich mit dem Religiösen verbindet. Auf Bedürfnisse zu reagieren, Wünsche aufzugreifen und ggf. Bedürfnisse dadurch zu wecken, daß ein latentes Verlangen und Sehnen erweckt, aufgegriffen und auf ein bestimmtes Produkt hingelenkt wird, das dessen Erfüllung verheißt - genau das macht das entscheidende Wesenselement der Werbung aus. Werbefachleute zeigen daher ein feines Gespür und eine hohe Sensibilität für das, was die Menschen tatsächlich bewegt. Sie verstehen "den Menschen zunächst keineswegs als ein Wesen, das auf materielle Erfüllung aus ist ${ }^{\prime \prime}$. Wo sie daher einschlägige und unverkennbar religiöse Elemente in ihre Arbeit einbeziehen, verraten sie viel über den Menschen. Sie deuten an, worauf sein Verlangen sich ausrichtet, ohne daß ihm dies bewußt sein muß. Werbung verheißt die Erfüllung eines Bedürfnisses, welches sie gezielt anspricht und dem sie quasi-religiösen Charakter zubilligt. Nur so läßt sich begreifen, warum sie in so reichem Maße die eingangs genannten religiösen Elemente einbringt.

\subsection{Die Sehnsucht nach letzter Gerechtigkeit im Kriminalfilm}

Nicht zu unterschätzen ist auch das religiöse Potential des unendlichen und scheinbar unverbrauchbaren Genres des Kriminalfilms. Daß er sich dauerhafter Beliebtheit erfreut, kann kaum verwundern. Er zehrt von einem urtümlich religiösen Verlangen des Menschen und entspricht ihm zugleich. Auf endgültige Gerechtigkeit in der Welt zu hoffen, auf den Sturz des Bösen zu setzen und dem Guten seinen Lohn und sein Recht zu sichern - das bleibt die drängende Sehnsucht und der insgeheime Wunsch eines jeden Menschen. Ihn erfüllt der Kriminalfilm auf die

7 Albrecht (Anm. 1) 48f (samt Abbildung).

8 A.a.O., 46. 
Weise des Medienreligiösen. Dabei vermittelt er unter eher moralischreligiöser Rücksicht auch die Botschaft, daß das Unrecht, die Gewalttat und das Böse sich nicht lohnen, daß sie deshalb zu meiden sind und dem Guten statt dessen der Sieg verheißen ist.

\subsection{Die Integration des fragmentarischen Lebens in Fernsehserien}

Auch Serien, Sendereihen und Mehrteiler haben ihren festen Ort im Angebot des Medienreligiösen, obwohl dies nicht auf den ersten Blick klar wird. Doch indem sie eine letztlich zusammenhängende Handlung segmentieren und in beinahe unendlicher Folge die dadurch gewonnenen Bruchstücke wieder zu einem Ganzen, eben zu einer kohärenten Abfolge in der Serie zusammenfügen, vermitteln sie dem Zuschauer damit, wenn auch eher unterschwellig, eine wahrhaft religiöse Botschaft, die dieser tief in sich hinein aufsaugt: Soviele Fragmente und Einzelstationen, soviele Szenen und Abbrüche das Leben auch haben mag - es fügt sich auch hier alles zu einem Runden und Ganzen, zu einer logischen Sequenz, zu einer letzten, alles miteinander verbindenden Einheit zusammen. Es ist die suggestive Vermittlung einer letztlich unteilbaren und garantiert von einem zum anderen fortschreitenden und alles miteinander verbindenden Ganzheit des Lebens, welche hier als eine Art persönlicher Sinnzuspruch das Religiöse in diesem Segment des Mediums Fernsehen ausmacht.

\subsection{Die "andere Seite" des Lebens in Fernsehshows}

Selbst die in großer Vielfalt angebotenen Shows kommen nicht ohne religiöse Botschaft aus. Ihr Ziel bleibt es, Menschen zu unterhalten, und zwar ohne moralischen Zeigefinger oder mit irgendeiner sonstigen Ambition und Anforderung. Doch indem solche Shows Selbstzweck sind, indem sie als Kehrseite zum Alltag das Leben von seiner heiteren Seite zeigen und genießen lassen, indem sie diesen Genuß des Lebens allen ermöglichen, die zuschauen, gewinnen sie den Charakter von Feiern, die eine neue Dimension von Welt und Leben eröffnen. Man kann sich seines Daseins freuen, unbeschwert lachen; man kann dasein und dasitzen, ohne - wie sonst im Leben - irgendeine besondere Leistung zu erbringen; man bekommt Unterhaltung und Freude in der Gegenwart geschenkt und fühlt sich dadurch wohl und beglückt. So klein und bescheiden, so verächtlich gering und banal dieses Glück auch anmuten mag - es gibt unendlich viele Menschen, die sich darauf mehr freuen als auf eine gemeinschaftliche Feier mit anderen und die dabei doch ähnliches empfinden. Was sie hier erleben, kommt für sie diesem anderen Glück einer Feier gleich. Dabei eignet diesem ShowErlebnis ein unverkennbar religiöser Zug, weil sich darin das andere des Alltags, das Nicht-Zweckhafte, das Nicht-Selbstgemachte, sondern das reine Geschenk der Freude am Dasein vermittelt und diese Erfahrung über alles hinausweist, was Menschen greifbar zur Verfügung haben und aus eigenen Kräften leisten können. 


\subsection{Träume und Traumerfüllungen in einschlägigen Produktionen}

Man wird das Medienreligiöse auch in den zahlreichen TraumProduktionen des Fernsehens erkennen dürfen. Die hier angezielte Ebene verbindet das eben über die Show Gesagte mit dem, was bereits für jene Züge der Werbung galt, die Himmel und Paradies in Anspruch nehmen. Menschen durch die Begegnung mit dem Fernen, dem Exotischen, mit dem Traum-Schiff am Traum-Strand unter schattigen Bäumen u.ä. zum Träumen zu bringen, sie auf diese Weise zu veranlassen, sich über ihre unmittelbar gegenwärtige Befindlichkeit hinaus Größeres, Schöneres ja sogar Paradiesisches als erreichbares Ziel und mögliche Erfüllung vor Augen zu stellen - das ist es, was solche Sendungen als Medienreligiöses produzieren und an-bzw. aussprechen. Sie geben einem anderen, einem Zukünftigen, einer Verheißung Raum, die die scheinbar unumstößliche Festlegung auf die Gegenwart und ihre engen Grenzen sprengt. Das Unmögliche erscheint nun möglich. Menschsein öffnet sich hier geradezu ins Unendliche, eben in unendliche Fernen. Damit integrieren und deuten diese Produktionen zugleich auf eine quasi-religiöse Weise den millionenfachen Aufbruch von Menschen in ferne Urlaubsländer. Sie machen sich das darin ebenfalls als ur-religiös artikulierende Verlangen und Sehnen nach dem zu eigen, was mehr und anders ist als der Alltag, die gewohnte Umgebung und die tägliche Sorge.

\subsection{Strukturierung der Zeit mittels fixer Programmpunkte}

Noch ein allerletztes, wohl das überraschendste Element des Medienreligiösen sei bedacht. Durch seine weite Verbreitung und seine ganz selbstverständliche tagtägliche Nutzung dient das Fernsehen und seine Programmstruktur einer unendlich großen Zahl von Menschen als jenes Medium, durch welches die Zeit maßgeblich strukturiert wird - eine Funktion, die seit frühesten Zeiten der Menschheit religiös verstanden wurde. Waren es früher Sonnenaufgang und Sonnenuntergang9, genauer: das Tageslicht und die Finsternis der Nacht sowie die Zeiten von Saat und Ernte, von Regen und Sonnenschein, die dem Leben seinen Rhythmus und seine Einteilung gaben, so hat das Christentum mit dem Morgen-, Mittag- und Abendläuten als Einladung zum Gebet, aber auch mit dem von ihm entfalteten Kranz von Festen der an sich gleichmäßig verlaufenden Zeit eine eigene, religiös begründete Struktur verliehen. Diese Grundfunktion des Religiösen ist aber offenbar zu einem Teil in das Medium Fernsehen abgewandert, von ihm übernommen und von vielen fraglos akzeptiert worden. Wochentage werden charakterisiert durch bestimmte Serien und Mehrteiler in meist regelmäßiger Abfolge und Wiederkehr. Die Winterzeit, also die Zeit außerhalb der immer

9 Dazu sehr schön und anregend Hermann Timm, Zwischenfälle. Die religiöse Grundierung des All-Tags, Gütersloh 1983, 23-36. 
länger währenden Sommerpause, wird zur Zeit der großen Samstagabendshows, die ebenfalls in periodischem Rhythmus ausgestrahit werden. Die Sommerzeit wird charakterisiert als die Zeit des Fernsehgartens, der mehr als vieles andere den eigentlichen Beginn bzw. das Ende des Sommers bezeichnet. Endlich aber findet der Abend seine Struktur und seine Bedeutung durch die großen Nachrichtensendungen, die - dem alten Ritual des Innehaltens zum Beten des Angelus oder auch dem den Tag zusammenfassenden Abendgebet täuschend ähnlich - jene Grundinformationen bereithalten, mit denen sich das Leben bestehen läßt. Es lag wohl nicht zuletzt an dieser besonderen Akzentuierung des Abends durch regelmäßige Fernsehsendungen, daß sehr rasch und geradezu zwanghaft auch das Frühstücksfernsehen hinzutrat. Ohne dies überzubewerten läßt sich in dieser Fernsehbegleitung bzw. schwerpunktmäßigen Strukturierung des Tagesablaufs eine Analogie zum Morgen- und Abendgebet feststellen, das hier gleichsam eine medienreligiöse Renaissance erleben darf.

\section{Die Herausforderung des Medienreligiösen in kritischer Sympa- thie anerkennen}

Was damit als vielfältige Gestalt und Gestaltung des Medienreligiösen klarer vor Augen steht, verdient keineswegs bloße Verachtung und Mißbilligung. $\mathrm{Daß}$ hier die Ebene des Ausdrücklich-Religiösen nicht erreicht wird, versteht sich von selbst. Doch sollte man sich sorgsam davor hüten, deshalb von einem säkularisierten Abklatsch, einer völligen Verwässerung und Banalisierung christlicher oder allgemeinreligiöser Elemente oder auch ganz undifferenziert von durchsichtiger Eindimensionalität und Kommerz zu sprechen. Dieses Medienreligiöse darf nicht nur als ein schlichtes Faktum hingenommen werden. Angesichts seiner erstaunlichen Verbreitung und der damit verbundenen Akzeptanz müssen gerade die christlichen Kirchen dieses Phänomen als eine echte Herausforderung begreifen, soviel Kritik es auch unter anderer Rücksicht verdienen mag.

\subsection{Respekt vor dem Medienreligiösen statt überheblicher Verachtung}

Üblich und weit verbreitet ist allerdings leider eine Haltung, die das Medienreligiöse zwar wahrnimmt, ihm aber jede Form von Anerkennung verweigert. Dabei verübeln die Kirchen nicht zuletzt die angebliche Profanisierung ihrer heiligsten Vollzüge. Als sprechendste Beispiele dienen dafür oft die ganz vom religiös-liturgischen Glanz zehrende Show "Traumhochzeit“, aber auch die offenbar der kirchlichen $\mathrm{Bu}$ - und Vergebungspraxis den Rang ablaufenden Sendung "Verzeih mir". Doch gerade diese beiden Produktionen machen deutlich, daß sich im Fernsehen elementare religiöse Fragen ${ }^{10}$, eben als das Medienreligiö-

10 Vgl. Wolfgang Fischer, Die Fernsehshow, in: Bieger (Anm. 1) 47-49. 
se, in veränderter Gestalt und sicherlich mit geringerer Tiefendimension als im kirchlichen Bereich stellen und behandeln lassen. Die Menschen erscheinen dafür keineswegs so unsensibel, wie eine ihr eigenes Versagen damit kaschierende, zugleich aber bloßlegende und vergeblich rechtfertigen wollende Theologie und Kirche behaupten. Sie sind vielmehr offenbar durchaus ansprechbar für solche Momente, auch der christlichen Religion, die an allgemein-religiöse Fragen und damit verbundenen Aspekten des Menschseins anknüpfen.

$\mathrm{Da} \beta$ in letzter Zeit vermehrt Kirchenmänner und auch Frauen der Kirche zu Titelfiguren von gern gesehenen Fernsehserien geworden sind und noch immer werden, zeigt dabei ein erstaunliches Verlangen nach solchen Menschen und Helfern, "die ihre Hilfe nicht an ServiceKategorien von Behörden, Betrieben, Banken festmachen, sondern an dem gelebten Glauben an Gott".11 Zumindest hier aber müßten die Kirchen höchst sensibel werden und erfreut wahrnehmen, wie sich - oft reichlich unverblümt - das diffuse Medienreligiöse in direkte Erwartungen und Erwartungshaltungen gegenüber den christlichen Kirchen übersetzt und darin verdeutlicht und artikuliert. Wie schon bei den früheren Beispielen gilt auch hier, daß hochnäsige Verachtung und dogmatisch angelegte Maßstäbe für solche Produktionen an der darin sich anzeigenden Wirklichkeit eines neuen religiösen Verlangens völlig vorbeigehen.

\subsection{Das Je-Größere in den Gestalten des Medienreligiösen}

\subsubsection{Bedürfnis-Überschuß in der Werbung}

Wendet man den Blick nochmals zurück auf die im ersten Schritt knapp umrissenen und auf ihre religiösen Dimensionen hin verdeutlichten Momente des Medienreligiösen, so zeigt sich hier eine reiche Palette von menschlichen Erwartungen, anders gesagt: ein Heilsverlangen, das alles andere als bloße Verachtung verdient, weil es die religiöse Dimension des Menschseins offenlegt und diese als keineswegs verschüttet darstellt. Werbung kann schließlich - so hat sich gezeigt - nur funktionieren, wenn Menschen sich mit dem nicht zufriedengeben, was sie augenblicklich sind und haben. Das Mehr-Sein- und MehrHaben-Wollen, die Sehnsucht über das Gegenwärtig-Faktische hinaus darf als die noch allgemeinste und ursprünglichste Bewegung des Religiösen gelten, auf die auch das Christentum selbst zweifelsfrei setzt und setzen muß. Dabei bleibt freilich kritisch zu vermerken, daß im Erwecken erheblicher Bedürfnisse die Werbung diese dann doch wieder reichlich handgreiflich und horizontalistisch zu befriedigen sucht. Anders verlöre sie schließlich ihren Sinn und das vorgestellte Produkt

11 So Reinhold Jacobi, Sendereihen und Mehrteiler, in: Bieger (Anm. 1) 36-38, hier 38 . 
seinen Markt und seine Käufer. Die Kommerzialisierung dieses religiösen Verlangens von Menschen und seine ökonomische Funktionalisierung anzuprangern, dabei aber die "kleinen Erfüllungen" als sprechende Symbole und zeichenhafte Gestalten der Verheißung von Größerem $\mathrm{zu}$ begreifen und $\mathrm{zu}$ deuten, wäre wohl die richtige Art, diese erste Gestalt des Medienreligiösen kirchlicherseits kritisch-sympathisch aufzugreifen.

\subsubsection{Letzte Fragen im Kriminalfilm}

Ähnliches gilt für den Kriminalfilm und seine religiöse Botschaft, daß letzten Endes die Gerechtigkeit siegt und die Welt in Ordnung bleibt, auch dann, wenn sie aus den Fugen geraten zu sein scheint. Die gelegentlich erstaunliche Sensibilität für die Verstrickung in die eigene Schuld und Untat und deren Tragik gewinnt manchmal eine solche religiöse Dichte, daß sie durchaus das Niveau für eine Anknüpfung und Überbietung in christlicher Verkündigung erreicht. In der Suche nach Ordnung und Gerechtigkeit fragen Menschen über das Medienreligiöse hinaus und zeigen sich damit offen für eine ebenso glaubhafte wie verständliche Auskunft über die letzten Fragen des Daseins.

\subsubsection{Der Wunsch nach ganzem und heilem Leben (Serien)}

Auch die Suche nach einem letzten Sinn und Zusammenhang des unendlich segmentierten eigenen Lebens, wie sie im Kontext der medienreligiösen Botschaft von Serien aufscheint, verdient sympathische Kritik. Gewiß lassen sich Menschen hier einlullen und suchen auf wenig tragfähigem Niveau die Erfüllung ihrer religiösen Bedürfnisse. Dennoch offenbart sich hier ein letzter Rest von Gespür dafür, daß menschliches Leben über sich hinausragt und mehr bedeutet, als die Zusammenfügung eines unendlichen Puzzles, welche niemals gelingt und woran der Mensch letztlich zerbricht.

\subsubsection{Die Suche nach Glück und Lebensfreude (Shows)}

Die andere Seite des Lebens, die im ersten Aufbruch dem Religiösen, dadurch aber auch dem Christlichen eine echte Chance gibt, charakterisiert die medienreligiöse Qualität der Shows. Es gehört zu den großen Torheiten unserer Zeit, daß gerade von christlicher Seite gegen solche leichte und triviale Form der Unterhaltung Front gemacht wird. Natürlich eignet ihr nicht die hohe Qualität von liturgischen Feiern. Doch helfen solche Shows offenbar dem sprach- und ausdrucksbehinderten Menschen der Gegenwart dabei, das Unverrechenbare, das Unverdienbare, das ohne eigenes Zutun mit Glück und Freude Beschenkende wenigstens ansatzweise zu erfahren. Sie lassen ihn so nicht völlig im Alltag und in seinen vielfältigen, meist als unbefriedigend empfundenen Rollen versinken und untergehen. Daß Leben sich lohnt, daß man lachen und sich freuen kann, trotz aller Widrigkeiten und trotz düsterer Perspektiven, bleibt eine ur-religiöse Botschaft und Zumutung. Nach christlicher Überzeugung ragt diese zwar noch weit über das Anschauen einer Fernsehshow hinaus, doch wird dadurch dieses kleine Glück weder unbedeutend noch madig gemacht. 


\subsubsection{Der Traum von einer neuen Welt}

Gleiches gilt von den Träumen, die das Fernsehen weckt und die das Sehnen des Herzens nach himmlischen und paradiesischen Orten und Zeiten wachruft. Gewiß verbindet auch hier das Medienreligiöse die damit erweckte ur-religiöse Sehnsucht sofort mit einer unbefriedigend anmutenden Erfüllung. Daß die im Medium bereits Wirklichkeit gewordenen Träume zerplatzen und doch noch Raum und Anlaß zu neuen Träumen bleibt, wird man sicherlich kritisch vermerken müssen. Doch selbst dann noch bleibt wichtig, daß es offenbar ein Potential an Träumen und Sehnsüchten gibt, deren sich der heutige Mensch keineswegs schämt, sondern denen er gerne und oft nachhängt. Solche Träume aufzugreifen, sie größer und tiefer, intensiver und geradezu grenzenlos werden zu lassen, bleibt eine Herausforderung des Medienreligiösen, der sich das Christentum kaum verschließen kann.

\subsubsection{Die Herausforderung der Zeit bestehen}

Schließlich und letztlich ist auch in der Suche des Menschen nach einer Strukturierung der Zeit mit Hilfe des Mediums Fernsehen ein religiöses Element festzustellen, das Beachtung verdient. Als einer, dem es aufgegeben ist, seine Zeit buchstäblich auszukaufen und auszunutzen, bleibt der Mensch darauf angewiesen, diese gleichmäßig fließende Zeit zu bewältigen, indem er sie in eine Ordnung und in einen gewissen Rhythmus bringt und sie so als den alles vernichtenden Moloch bändigt. An die Kirchen ergeht aus dieser medienreligiös gearteten Bewältigung der Zeit die Frage, welche Hilfe sie dem Menschen bei dieser Lebensaufgabe anbieten, ob sie diese als eine echte Herausforderung begreifen und wie sie darauf $\mathrm{zu}$ antworten gedenken. Daß alte Antworten auch hier nicht genügen, daß ein bloßer Rekurs auf Morgenund Abendgebet oder gar eine Wiederbelebung des Angelus nicht genügen kann, versteht sich dabei von selbst. Ebensowenig darf man verschweigen, daß eine vom Medium allein bedingte Strukturierung der Zeit sich auf Dauer kaum als tragfähig erweisen wird, zumal es ihr an inhaltlicher Füllung und daraus erst entspringender Legitimation fehlt.

\section{Das Medienreligiöse in der Liturgie selbstkritisch aufgreifen und überbieten}

Damit haben diese kritischen Sichtungen der anstehenden Herausforderung bereits unversehens zum entscheidenden dritten Schritt übergeleitet. Die eingangs behandelte Titelfrage hat sich dabei durch eine genauere Betrachtung des Medienreligiösen und der daraus sich ergebenden Ansprüche an die Kirchen und ihre Theologie entscheidend verwandelt. Nicht mehr die Überbietung von Fernsehshows durch die Liturgie erweist sich nun als das zentrale Problem. Vielmehr stellt sich die viel weiter reichende Frage, ob und inwiefern die christliche Liturgie - zumindest grundsätzlich - fähig und bereit ist, die im Medienreligiösen sich artikulierenden oder auch nur versteckt andeutenden religiösen 
Bedürfnisse der Gegenwart ebenso selbstkritisch wie produktiv aufzugreifen und so die vielbeschworenen "Zeichen der Zeit"12 zu erkennen und sich damit als wahre Liturgie des Volkes bzw. von Volkskirchen zu zeigen. So konkret die damit bezeichnete Aufgabe auch gemeint, vor allem aber anzugehen ist, so prinzipiell und allgemein, damit aber keineswegs abstrakt, müssen die wenigen Hinweise geraten, die sich mit der Beantwortung dieser tiefer und weiter reichenden Frage befassen.

\subsection{Rückbesinnung auf die symbolische Gestalt christlicher Heilsvermittlung}

Der Herausforderung durch die Werbung sollte die Liturgie nicht zuletzt mit der klaren Rückbesinnung darauf begegnen, daß auch sie die letzte Erfüllung menschlicher Bedürfnisse, besser: der menschlichen Bedürftigkeit, nur im Symbol, nur im Zeichen vermitteln kann. ${ }^{13}$ Dabei darf sie, mehr als bisher, auf die Selbstevidenz der grundlegenden Symbole setzen. Sie sollte diese nicht durch endlose und oft überflüssige Katechese ihrer Eigenmächtigkeit und damit ihrer (an den vielen Beispielen aus der Werbung sichtbaren) eigenen Stringenz und Aussagekraft entkleiden und so entwerten. Der Mut zum Symbol von Wasser und Feuer, Licht und Brot läßt sich gerade im Blick auf das Medienreligiöse wieder neu gewinnen. Wie kein Showmaster das Anzünden von Wunderkerzen oder das Aufblinken von Taschenlampen dadurch um seine unübertreffbare Eigenwirkung bringen würde, daß er dessen Sinn, nämlich den Ausdruck von Freude, Gemeinschaft, Übereinstimmung, Zustimmung, Lebensglück, lichter Helle und Heiterkeit des Lebens wortreich-geschwätzig erklärte, so darf auch die Liturgie Zutrauen fassen zu ihren Zeichen, die überdies meist ur-religiöse Symbole sind. Wenn nicht alles täuscht, gibt es auch in der Gegenwart noch bzw. gerade wieder ein abgrundtiefes Mißtrauen gegenüber der großen Offenheit und Randunschärfe alles Symbolischen, das sich niemals völlig auf den Begriff bringen läßt. ${ }^{14}$ Es steht damit unter streng

12 Diese wegweisende, jedoch selten ernstgenommene Formel für eine zeitgemäße Hermeneutik von Glaube und Welt fand das Zweite Vatikanische Konzil in seiner Pastoralkonstitution "Gaudium et spes" Art. 4.

13 Über neuere Arbeiten zu Sinn und Bedeutung des Symbols vgl. meine Lit.berichte: Das Sakrament als Symbol, in: Franz Böckle u.a. (Hg.), Christlicher Glaube in moderner Gesellschaft, Enzyklopädische Bibliothek 28, Freiburg 1982, 122-150; "Feier" (Anm. 2); Symbolwirklichkeit und Sakrament. Ein Literaturbericht, in: Liturgisches Jahrbuch 40 (1990) 26-52; dazu auch die kürzlich erschienene Münchener Habilitationsschrift von Heribert Wahl, Glaube und symbolische Erfahrung. Eine praktisch-theologische Symboltheorie, Freiburg 1994; dort 525-589 auch zur symbolischen Dimension von Liturgie und Sakrament.

14 Vgl. dazu meine grundsätzlichen Erwägungen in: "Gedachte Liturgie“ als Mystagogie. Überlegungen zum Verhältnis von Dogmatik und Liturgie, in: Eberhard Schockenhoff und Peter Walter (Hg.), Dogma und Glaube. Bausteine für eine theologische Erkenntnislehre. Festschrift für Bischof Walter Kasper, Mainz 1993, 213-234. 
dogmatischer Rücksicht natürlich stets unter dem Verdacht, eine gehörige Portion Unglaube zu bewirken oder gar zu legitimieren. Daß diese urtümlich rationalistische Angst vor der Lebensfülle und Inhaltsdichte des Symbols noch immer vorherrscht, bringt die Liturgie gegenüber dem hier ganz unbefangen agierenden Medienreligiösen erheblich ins Hintertreffen. Dabei muß diese Wendung zum Symbolischen bis in die Sprache hineinreichen. Gerade so werden jene Menschen aufmerksam und aufnahmefähig, die sich zunächst mit dieser Art des Medienreligiösen begnügen und darauf verstehen, die aber durchaus ansprechbar wirken und daher zu weiteren Schritten auf dem Weg des Glaubens bereit sein könnten.

Die Führung und Weggeleitung durch Symbole und symbolische Rede muß dabei keineswegs als Verführung verdächtigt werden. Offenbar versteht der Mensch unserer Tage symbolisches Geschehen und symbolgeladene Sprache gerade im Blick auf latent oder offenkundig religiöse Gehalte viel besser und leichter als schwergewichtige lehrhafte Ausführungen oder katechetische Unterweisungen. Daß Christsein aus dem Vollzug symbolischer Handlungen lebt und darin eines seiner erkennbarsten und wichtigsten Wesensmomente liegt, gehört immerhin zu den herausragenden Entdeckungen der Theologie im 20. Jahrhundert. ${ }^{15}$ Wo die Kirche und ihre Theologen sich zu lange geziert haben, hat eine findige und clevere Schar von Werbe- und Medienexperten längst erkannt, was an der Zeit ist. Symbolischer Ausdruck und symbolische Artikulation bleiben nicht nur dem Säkularreligiösen eigen. In dieser Struktur begegnen sich das allgemeine wie das spezifisch christliche Religiöse.

Das gilt vor allem für die Liturgie. Ihr Reichtum an Symbolen und ihr eigenes Wesen als symbolische Handlung macht sie fähig, dem Religiösen überhaupt einen genuinen und legitimen Platz zu geben und damit ganz nahe. bei den Menschen zu sein. Daß die Vielschichtigkeit und Vieldeutigkeit der Symbole deren religiöse Bedeutung auch offen sein läßt für mehr und für Größeres, gibt ihnen erhebliches Gewicht. Angesichts einer vielfältigen Wiederkehr des Symbols kann das Christentum jedenfalls nicht die Flucht vor dem Symbol ins reine Wort hinein ergreifen oder gar predigen. So verliert es endgültig die Rückbindung an jenes Moment von Religion, das sich - wenn auch auf völlig verschiedene Weise - im Medienreligiösen artikuliert und auch im christlichen Glauben fundamental wichtig bleibt.

15 Dies gilt vor allem für die Mysterientheologie Odo Casels, die sich unter dieser Rücksicht als langfristig besonders wirksam und aktuell erwiesen hat. Für ihn bildet den Mittelpunkt des Christentums die kultische Handlung, der Vollzug der "Mysterien", also die Liturgie als Handlungsganzes. Vgl. dazu meine Arbeiten: Theologie (Anm. 2); Erneuerung der Sakramententheologie im 20. Jahrhundert. Ein Blick auf die Anfänge bei Romano Guardini und Odo Casel, in: Liturgisches Jahrbuch 37 (1987) 17-41; Christentum (Anm. 3); Gegenwart (Anm. 2; Lit.). 


\section{2 Öffnung des Medienreligiösen für die Verheißungen des Glaubens}

\subsubsection{Christliche Liturgie als Ort unverkürzten Lebens}

Dieser Gedanke läßt sich in anderer Wendung noch vertiefen und ausarbeiten. Indem die Werbung Bedürfnisse weckt und zugleich deren Erfüllung verspricht, kommt sie der Liturgie durchaus nahe. Diese setzt letztlich auf ein alles überragendes Bedürfnis der Menschen und erweist sich dabei als fähig, viele andere und damit als vorläufig anmutende Elemente jenes Religiösen, das sich in den Medien findet, aufzugreifen, einzubeziehen und überbietend zu präsentieren.

Von sich aus will Liturgie in symbolisch-gemeinschaftlicher Feier, in festlich-frohem, den Alltag überschreitenden Miteinander von Menschen etwas von dem vermitteln, was christliche Verheißungswirklichkeit ausmacht - den Himmel als die vollendete Gemeinschaft der Menschen mit Gott und untereinander. ${ }^{16}$ Breiter und höher kann der Schirm der Hoffnungen und Erwartungen nicht ausgespannt werden, der sich im gesamtsymbolischen Geschehen der Liturgie wie in ihren einzelnen Symbolen und Symbolhandlungen auftut. Ihr Auftrag ist es, die Sehnsucht nach einer neuen und anderen, einer friedlichen und glücklichen Welt immer neu zu wecken und wachzuhalten. In ganz anderer Weise als Hollywood, als die Werbung oder als Traumserien stellt sich die Liturgie daher als eine Art "Traumfabrik" dar, die Gottes Traum mit dieser Welt und den Menschen vorstellt und zum Mit-Träumen einlädt. Sie verspricht hier und jetzt nur Erfüllung im Fragment, für die Zukunft aber die Vollendung und Erfüllung auch der höchsten menschlichen Träume. Sie verachtet keineswegs das kleine Glück des Lebens in seiner vielfältigen Gestalt, sondern bringt es in Lob und Dank mit ein.

Sie weiß aber auch darum, wieviel noch aussteht von diesem letzten Glück, welche Hindernisse sich auf diesem Weg auftürmen, welche Schuld und welches Versagen das Ziel vor Augen verschwimmen und in weite Ferne rücken lassen, und $\mathrm{da} \beta$ am Ende zunächst anstelle offenkundiger Erfüllung der scheinbar alles vernichtende Tod steht. Auch dies, und gerade dies, spart die Liturgie nicht aus und geht daher ehrlicher um mit dem Menschen als die Werbung, die buchstäblich das Blaue vom Himmel herunter verspricht und dabei lügt. Im christlichen Gottesdienst haben Klage und Zweifel, Bitte und Fürbitte ihren festen Ort, weil die Vollendung des Menschheitstraums nicht dem Menschen selbst zugetraut, sondern von Gott erwartet wird. Ebenso

16 Vgl. dazu meine knappe Skizze: Der Himmel auf Erden. Zum Sakrament der Eucharistie, in: Jürgen Hoeren (Hg.), Wo Gott uns berührt: Der Lebensweg im Spiegel der Sakramente, Freiburg 1993, 41-57; ergänzend auch: Der Himmel - eine verlockende Zukunft? in: M. Plate (Hg.), Engagierte Gelassenheit. Impulse und Orientierungen für Zukunft aus dem Glauben, Freiburg 1978, 81-86. 
aber gibt die Liturgie den Mut und die Freiheit, das eigene Versagen, Schuld und Versäumnis an Gutem offen zu bekennen und dafür einzustehen, weil niemand daran scheitern und keiner sich dafür verstecken muß. Selbst die Trauer braucht nicht außen vor zu bleiben, noch muß sie zu kurz kommen. Schmerz und Tränen dürfen sein, weil erst am Ende klagloses Glück steht und Gott selbst jede Träne abwischen wird (vgl. Offb 21,4f), während diese Weltzeit noch von Gebrechlichkeit, Krankheit und Tod gezeichnet bleibt.

\subsection{2 Überbietung des Medienreligiösen in der Liturgie}

Diese vielschichtige Botschaft vermittelt die Liturgie keineswegs abstrakt oder aber allein im verkündeten Wort. Sie vollzieht sie in einer ebenso sprechenden wie mitreißenden Handlung, die der Show zumindest darin nahekommt, daß auch sie den Alltag kontrastiert und das Leben zum Fest und zum Genuß werden läßt, ohne daß der Mensch dafür etwas leisten, einbringen oder sich besondere Mühe geben müßte. Liturgie soll die Freude am Leben, geschenkt aus dem Glauben, zu reinstem Ausdruck bringen - keineswegs aber will sie den Alltag und die von Alter und Geschlecht, von Rang und Rolle, von Herkunft und Bildung bestimmte Unterscheidung zwischen Menschen widerspiegeln oder fortsetzen. Sie stellt dem eine andere Wirklichkeit und eine neue Welt entgegen, die zwar in manchem einer Show ähnlich sein mag. Doch damit verbindet sie den tiefsten und letzten Ernst, denn die liturgische Zusammenkunft und Feier steht stets mit dem ersten Wort und Zeichen unter dem Kreuz Jesu Christi und vollzieht sich ausdrücklich im Namen des dreifaltigen Gottes, des Vaters, des Sohnes und des Heiligen Geistes. ${ }^{17}$ Gegen die vielfach übliche freundliche Begrüßung am Beginn des Gottesdienstes soll damit nichts gesagt sein. Sie holt vielmehr den ab, der dieses Ritual aus dem Alltag, aber auch aus der medienreligiös gearteten Show kennt. Doch verschweigen darf die Liturgie nie, daß in aller festlicher Freude am Dasein hier ein anderer, tiefer Ernst mitschwingt, der den einzelnen zu wahrem Glück und zu reifer Freude hinführen will, in der auch die Schattenseiten des Daseins noch ihren Platz haben, ohne daß sie die Freude vergällen. Nichts gegen eine Liturgie, deren Anliegen es bleibt, daß die Menschen sich wohlfühlen im gottesdienstlichen Raum, denn nur so können diese "sich mit ihren Lebensinteressen in sie einbringen“.18 Doch man tut gerade denen, die bewußt, oft genug aber unbewußt, mehr suchen als nur eine Fortschreibung oder gar einen Abklatsch des Medienreligiösen, keinen guten Dienst, wenn man den umgreifenden Sinnhorizont des Gottesdienstes im unklaren beläßt und verschweigt, daß christliche Liturgie stets Gedächtnis des höchsten und letzten Ernstfalls von Tod und Auferstehung Jesu Christi meint.

\footnotetext{
17 Dies habe ich breiter ausgeführt in: Der Gottesdienst (Anm. 3), sowie: Das Menschen-Mögliche geschehen lassen. Vom Sinn der Liturgie, in: Hirschberg. Monatsschrift des Bundes Neudeutschland 48 (1995) 434-445. 
Dann aber gewinnt alles, so ähnlich es den Elementen und Strukturen gelungener Fernsehunterhaltung auch sein mag, einen anderen Ort und eine neue Bedeutung. Die vielfältigen Bruchstücke, das Fragmentarische und Unvollendete des eigenen Lebens kann sich hier widerspiegeln und doch zur Ganzheit finden. Möglich wird dies im Gedächtnis des am Kreuz mitten aus dem Dasein gerissenen Jesus, dem Gott in der Auferweckung sein Leben auf wunderbare Weise rund und ganz hat werden lassen. Der große Zusammenhang des Lebens, den Serien und Sendereihen medienreligiös suggerieren, wird hier grundlegend und umfassend versinnbildet und $\mathrm{zu}$ einem fundamentalen Symbol des christlichen Glaubens erhoben. Gelungene Liturgie kann und muß demnach das Fragmentarische des jeweiligen Lebens aufgreifen und jenen überragenden Zusammenhang vorstellen und herstellen, also wirksam werden lassen, den sie als ihre ureigene Verheißung vermittelt und in das Leben der Menschen einbringt. Indem sie das Gedächtnis des als Verbrecher endenden Jesus mutig begeht und damit ebenso einer Geschichte der Sieger wie dem scheinbaren Triumph derer widerspricht, die das Böse tun, nimmt sie die medienreligiöse Botschaft jedes Kriminalfilms in einzigartiger Weise auf und läßt dem Sieg des Bösen gegenüber dem alles in Recht und Gerechtigkeit richtenden und ordnenden Gott keinen Raum mehr. Weil die Liturgie zudem das "ganz andere" einer Traumwelt in recht ausdrücklicher, nämlich symbolischsinnenhafter Weise vermittelt und vor allem den Traum von einer neuen Menschheit und einer anderen Welt schon momenthaft im Vorgriff Wirklichkeit werden läßt, überragt sie die schalen Traumerfüllungen der Medien bei weitem, ohne dabei deren durchaus legitime und wichtige religiöse Dimension außen vor zu lassen. Auch hier gilt das Prinzip der Uberbietung, das diesen Ausgangs- und Anknüpfungspunkten jedes Recht bewahrt, dabei aber selbstredend nicht stehenbleiben kann.

\subsection{Praktische Anregungen und Konsequenzen}

Um all das zu leisten wird die Liturgie nicht einfach bleiben können wie sie ist. "In den Gottesdiensten müssen die Menschen wieder stärker das Gefühl gewinnen können, daß sie vorkommen mit ihrem eigenen Erleben und ihren eigenen Fragen, sie sich einbringen können. Eine ästhetisch gelungene Ausdrucksgestalt eigenen Erlebens sollte der Gottesdienst sein." 19 Die Liturgie muß sich also tief hinabbeugen, ohne sich dabei zu verkrümmen, um die Menschen mit ihrem im Medienreligiösen sich spiegelnden religiösen Verlangen zu erreichen, einzuladen, zum Mitmachen zu motivieren. Dabei wird sie zugleich eine partielle Distanzierung aushalten müssen. Sie wird sich um mehr Einfachheit und Schlichtheit, um die Durchsichtigkeit auf ihre prägende Grundge-

19 A.a.O., 50f; vgl. dazu nochmals Martin (Anm. 3) und grundsätzlich meine Ausführungen: Liturgie und Menschsein (Anm. 3); Christlicher Gottesdienst (Anm. 3). 
stalt bemühen müssen und dabei durchaus bei den Medien in die Schule gehen können.

\subsubsection{Hinweise zur Gestalt der Gottesdienste}

Fernsehshows leben von festgefügten Riten und gewinnen dadurch ihre Attraktivität und Plausibilität. Deshalb dürfte es langfristig ein kaum verheißungsvoller Weg, eher schon ein Irrweg sein, wenn man die Gottesdienste zum Experimentierfeld und zur Spielwiese freier Kreativität und Spontaneität geraten läßt. Eher schon überzeugend und zukunftsträchtig wirken würde, gerade im Blick auf die Shows, die schnörkellose, gekonnte, sympathisch-gewinnende und damit überzeugende Durchführung und Organisation der liturgisch-rituellen Feier. Dabei ist auch daran zu erinnern, wieviel Probenarbeit in solche Shows investiert wird und sich dann auch auszahlt. Man geht kaum fehl in der Annahme, daß die praktische Durchführung der Liturgie sich daran durchaus ein Beispiel nehmen könnte. Souverän und durchaus gekonnt muß der Umgang mit den Riten sein und wirken, nicht gezwungen, sondern so spielerisch und selbstverständlich, daß dahinter die harte Arbeit der Vorbereitung völlig verschwindet, ohne daß sie dadurch überflüssig wäre. $\mathrm{Ob}$ die konkreten christlichen Gottesdienste dieser Norm und Vorgabe immer standzuhalten vermögen, sei dahingestellt, durchaus aber bezweifelt.

Das gilt noch für ein weiteres Phänomen der Fernsehshows. Sie kennen die Einstimmung des Publikums, das niemals ohne gezielte Vorbereitung in die ausgestrahlte Sendung hineingeht. „Aufwärmphasen" verdienen keineswegs Spott und Hohn. Sie dienen auch dem durchaus liturgisch zu verstehenden - "transitus" in die andere Welt der Show bzw. der Feier. Gerade der heutige Mensch braucht solche Übergänge und Einstimmungen, und vieles deutet darauf hin, daß gerade an dieser Stelle eine entscheidende Schwäche der Gottesdienste liegt. Sie beginnen offenbar zu unvermittelt; es fehlt jene Einstimmung und Einübung, die kollektive Gemeinschaft bereits im Vorgriff herstellt. Das kann durchaus schlicht geschehen, etwa mit einem meditativen Orgelspiel, mit einer freundlichen wechselseitigen Begrüßung der Nachbarn, mit einer Vorstellung des Gottesdienstleiters oder sonstiger Hauptakteure der heiligen Handlung, mit dem Einüben eines Liedes oder eines Gottesdienstelements, aber auch mit der Einladung zu einer gemeinsamen Besinnung noch vor Beginn der eigentlichen Liturgie, die dieser dann den Weg bereitet. Auch auf diese Weise könnte es zu einer "Steigerung der Erlebnisintensität “20 kommen, die dem christlichen Gottesdienst keineswegs schlecht anstünde.

Endlich wäre auch jener Eigentümlichkeit des heutigen Menschen mehr Rechnung zu tragen, die dieser ebenfalls in der Medienwelt gerne

20 A.a.O., 50. 
und bewußt ausübt - dem Auswählen und der nur teilweisen Partizipation. ${ }^{21}$ Kein Alles-oder-Nichts-Standpunkt dürfte daher auch in der Liturgie gefragt sein, sondern das gelassene Ertragen einer gewissen Auswahlmentalität. Dabei kann man darauf verweisen, daß das Ganze des Glaubens und auch der Liturgie stets auf den Schultern aller und niemals auf denen eines einzelnen ruht, so daß er sich zu Unrecht oft genug überfordert fühlt. Die lebendige Erfahrung, bedingungslos und vorbehaltlos angenommen zu sein als einer, der nach Kräften - und zwar nur nach seinen eigenen Kräften, keineswegs aber nach einer starren Norm und Vorschrift - dabeisein will, sollte für jeden Teilnehmer am christlichen Gottesdienst möglich und grundlegend sein. Dies ist um so mehr begründet, weil sich in diesem Symbol die unbedingte Annahme aller durch Gott in Jesus Christus verdichtet und zugleich realisiert.

\subsubsection{Liturgie als konkrete Ermöglichung religiös strukturierter Lebens-Zeit}

Noch ein letztes Moment bleibt anzufügen, worin die Liturgie gerade in Begegnung mit dem Medienreligiösen und dessen Überbietung lernen könnte. Die Strukturierung der Zeit durch Feste und Feiertage, durch Sonntage und Wochentage bleibt nach wie vor eine wichtige Leistung des Christentums und seiner gottesdienstlichen Feiern.22 In der Pluralisierung religiöser Lebenswelten ist diese Grundorientierung allerdings oft genug verlorengegangen und - wie gezeigt - gelegentlich vom Fernsehen übernommen worden. Allerdings bleibt auch hier die Liturgie zu neuen und unkonventionellen Wegen unter Inanspruchnahme ihrer ureigenen Möglichkeiten aufgerufen. Daß gerade sie - wie der christliche Glaube ganz grundsätzlich - im Dienst an den Menschen steht, dem Gott selbst sich in Jesus Christus verbindlich zugewandt und verbunden gezeigt hat, sollte dabei nicht vergessen werden. Daher muß der Gottesdienst und die mit ihm verbundene Strukturierung der Zeit auch dem heutigen Menschen noch Hilfestellung und Orientierung geben können.

21 Vgl. dazu ebd., aber auch Volker Drehsen, Wie religionsfähig ist die Volkskirche? Sozialisationstheoretische Erkundungen neuzeitlicher Christentumspraxis, Gütersloh 1994 (Lit.), bes. 174-198, 250-285, 313-345 (Lit.), sowie Höhn (Anm. 1), bes. 108-138 (Lit.).

22 Das weitaus Beste dazu findet sich noch immer bei Peter Cornehl, Christen feiern Feste. Integrale Festzeitpraxis als volkskirchliche Gottesdienststrategie, in: Pastoraltheologie 70 (1981) 218-233; ders., Zustimmung zum Glauben. Eine Besinnung auf den Sinn der Feste und Feiertage, Pastoraltheologie 74 (1985) 410-425. Vgl. auch meine beiden kleinen Skizzen: Aus Festen leben. Religionsgeschichtliche und grundsätzliche Überlegungen, in: Erbe und Auftrag 68 (1992) 293-311; Vom Sinn christlicher Feste. Geschichtliche und liturgietheologische Hinweise im Blick auf Weihnachten und Epiphanie, in: Erbe und Auftrag 58 (1992), 452-470, sowie den zugehörigen Lit.bericht: Fest und Feier (Anm. 2). 
Das bedeutet zunächst einmal einen Verzicht auf jede Art von Überforderung, gerade bei der Feier jener christlichen Feste und Feiertage, die für viele, nur locker mit der Kirche verbundene Menschen noch immer fixe Orientierungsmarken abgeben. Dies gilt vor allem für das Weihnachtsfest, das sich im Laufe unseres Jahrhunderts vor allem mit vielfältigen gesamtkulturellen Bedeutungsgehalten verbunden und angereichert hat. „Das Deutungspotential zur Interpretation des Sinnes, den das Weihnachtsfest für die Menschen gewinnen kann, kann die Kirche nicht unter Absehung von den Deutungsleistungen zum Zuge bringen, die von den Menschen am Leitfaden der Konsumkultur, der Heiligung der Familie, der Mythisierung von Kindheit selbsttätig erbracht und von sekundären religiösen Institutionen des Kulturbetriebes verwaltet werden. Die Kirche kann sich als Ort religiöser Deutungskultur nur behaupten, indem sie diese alltagsweltlich verankerten und intermediär bereits abgestützten Deutungsleistungen ihrerseits aufnimmt, um sie in ihren auf erfülltes, gelingendes Leben gehenden Motiven zu erhellen, besser zu begreifen. Die Kirche muß versuchen, diese Motive mit den tradierten Deutungszuschreibungen des Christentums, also mit der Geschichte von der Geburt des Erlösers, zu konfrontieren, um die religiösen Motive, die die Menschen mit diesem Fest verbinden, in ihrem vielfach verdeckten Sinngehalt tiefer zu legen. ${ }^{\text {"23 }}$

Ähnliches ließe sich für das Neujahrsfest sagen, wobei der katholischen Kirche hier unerbittliche Selbstkritik gut anstände. Angesichts eines Tages, an dem Menschen dieser Kultur eine der tiefsten Zäsuren zur Strukturierung der Zeit erfahren und mit allen nur erdenklichen Mitteln zu bestehen und zu feiern versuchen, geht die Liturgie des 1. Januar souverän an sämtlichen damit verbundenen religiösen Momenten vorüber und feiert das „Hochfest der Gottesmutter Maria". ${ }^{24} \mathrm{DaB}$ es - paradox genug - im Rahmen der Eucharistiefeiern "in verschiedenen öffentlichen Anliegen“ dann tatsächlich eine solche "Zum Jahresbeginn" gibt, macht alles nur noch schlimmer. ${ }^{25}$ Genau so darf sich Liturgie nie gestalten, will sie das Medienreligiöse integrieren und damit zugleich in kritischer Überbietung überwinden.

Allerdings gibt es auch gelungene Beispiele solcher Ermöglichung einer religiösen Strukturierung der Lebens-Zeit. Eines hat das Jahr 1995 mit einer ebenso überraschend wie erfreulich intensiven Feier des evangelischen Buß- und Bettages gebracht. Offenbar ist es den evangelischen Kirchen und Christen trotz der Streichung des staatlichen Feiertags gelungen, den Festgehalt dieses Tages und den Sinn dieser

23 Gräb (Anm. 4) 51

24 Die Feier der Heiligen Messe. Meßbuch. Für die Bistümer des deutschen Sprachgebietes. Authentische Ausgabe für den liturgischen Gebrauch. Kleinausgabe, Einsiedeln 1978, 50f.

A.a.O., $1061 \mathrm{f}$. 
liturgischen Feier klarer vor Augen zu stellen und im Bewußtsein vieler zu verankern. In Anknüpfung an jenes allseits anerkannte und bis in den Bereich des Medienreligiösen reichende Bewußtsein von Schuld und Vergebung ist es in diesem Fall anscheinend geglückt, die einzigartige christliche Verdichtung dieses ur-religiösen Moments eindringlich und wirkungsvoll nahezubringen und diesen Tag als tiefen Einschnitt und Wendepunkt in der zerfließenden Zeit festzumachen. Ähnliches mag für ein anderes Beispiel gelten, das aus den früheren adventlichen Mettenfeiern im katholischen Bereich mit Blick auf junge Christen und in Anerkennung des Gewichts sozialer Wirklichkeit wie der Arbeitswelt insgesamt eine liturgische "Frühschicht" bzw. "Spätschicht" hat entstehen lassen, deren Attraktivität und Sinn unmittelbar einleuchten. Das gegen das normale Zeitempfinden angehende Phänomen der Schichtarbeit wird hier aufgegriffen und liturgisch konkretisiert und konfrontiert in einer Feier, die die Zeit buchstäblich anhält, Unterbrechung ermöglicht und Besinnung erlaubt.

Für eine ähnliche wünschenswerte Zeitgenossenschaft der Kirchen und ihrer Liturgie gäbe es noch eine Fülle weiterer Beispiele zu nennen. Eines mag für viele stehen, wenngleich es negativ ist. Während die christliche Begleitung der Kranken auch liturgische Gestaltung kennt, gibt es in den christlichen Kirchen bis heute kein irgendwie geartetes herausragendes liturgisches Geschehen, das den entscheidenden Überschritt vom aktiven Berufsleben in den Ruhestand begleitet und die religiösen Dimensionen dieses gewichtigen Lebenseinschnitts zu deuten weiß. Mag hier sogar das Medienreligiöse versagen - eine solche Zäsur bringt religiöse Fragen oder zumindest ins Religiöse hineinspielende Probleme mit sich, die auch in liturgischer Feier aufgegriffen werden könnten und sollten.

Mit diesen Hinweisen ist das eingangs genauer umrissene Thema noch keineswegs erschöpfend behandelt. Dennoch dürfte zumindest in groben Umrissen deutlich geworden sein, daß und wie die Kirchen mit ihrer Liturgie auf die Herausforderungen durch das Medienreligiöse zu reagieren und $\mathrm{zu}$ antworten hätten. $\mathrm{Daß}$ dieser letzte, zugleich entscheidende Schritt die unvoreingenommene Wahrnehmung der besonderen religiösen Zeitstruktur ebenso voraussetzt wie deren sympathisch-kritische Würdigung, hat sich dabei zu Genüge gezeigt. Auch hier gilt: Wer die richtigen Fragen kennt oder zu stellen weiß, verfügt bereits über die halbe Antwort. Da Kirche und Theologie genau dieses bislang noch weitgehend versäumt haben, wird wohl auch die richtige Antwort noch etliche Zeit auf sich warten lassen. 
SUMMARY: Lithurgy - the better show? Media-religion as challange for the churches

There is a lot of not church-bound religiosity found in all kinds of media, especially in TV. Starting out from publicity to crime-movies and series as well as shows, media-religious elements are to be found. The churches and theology have to register carefully this religious elements in TV and even beat them. Doing so, lithurgy may trus more than before on its own symbols and symbolic actions. Actually, Christian preaching of salvation includes itself a symbolic figuration. Moreover, Christian lithurgy proofs to be a place and feast for a total life without shortennings. Thus, lithurgy integrates and exceeds the temporary fulfilment in the media-religious field.

RÉSUMÉ: Liturgie - Le meilleur spectacle? „Le religieux dans les médias" comme défi à l'église

La religiosité en dehors de l'église se trouve de façons multiples dans les médias, surtout à la télévision. De la publicité en passant par les films policiers jusqu'aux feuilletons et aux spectacles, on distingue des éléments de ce qui est "religiuex dans les médias". L'église et la théologie doivent tout d'abord percevoir avec attention cette dimension religieuse de la réalité médiatique. La liturgie doit saisir de tels moments d'éléments religieux dans les médias et surenchérir. En cela, elle doit faire plus confiance que jusqu'à présent à la force de persuasion de ses symboles et de ses actions symboliques. Finalement, la procuration du salut est elle-même uns propre forme symbolique. De plus, la liturgie chrétienne se trouve être l'endroit et la fête d'une vie integrale et non-raccourcie. Elle intègre et dépasse ainsi les accomplissements momentanés des „élements religieux dans le médias".

RESUMEN: La liturgia - ¿Un buen Show? La manifestación de lo religioso en los medios como desafío para las iglesias

La religiosidad extraeclesial se encuentra presente en sus más diversas manifestaciones también en los medios y especialmente en la televisión. Desde la publicidad, pasando por las películas policiales hasta las serie y shows se muestran elementos religiosos. La iglesia y la teología deben tomar consciencia de esta dimensión de la realidad en los medios. La liturgia debe hacer suyo y mejorar estos momentos de la realidad religiosa de los medios. En esto puede confíar en la fuerza de convioción de sus símbolos y de sus ceremonias simbólicas. Por último, la transmisión de la buena nueva cristiana hay de adquirir una forma simbolica. Además la liturgia cristiana se muestra como lugar y celebración de una vida plena y no cercenada. Integra y supera de esa manera la satisfacción circunstancial de los deseos que se da en la religiosidad de los medios. 